\title{
HYBRID CONTROL OF ELECTRIC VEHICLE LATERAL DYNAMICS STABILIZATION
}

\author{
Khatir Tabti - Mohamend Bourahla - Lotfi Mostefai
}

\begin{abstract}
This paper presents a novel method for motion control applied to driver stability system of an electric vehicle with independently driven wheels. By formulating the vehicle dynamics using an approximating the tire-force characteristics into piecewise affine functions, the vehicle dynamics cen be described as a linear hybrid dynamical system to design a hybrid model predictive controller. This controller is expected to make the yaw rate follow the reference ensuring the safety of the car passengers. The vehicle speed is estimated using a multi-sensor data fusion method. Simulation results in Matlab/Simulink have shown that the proposed control scheme takes advantages of electric vehicle and enhances the vehicle stability.

K e y w or d s: electric vehicle, hybrid model predictive control, yaw moment control
\end{abstract}

\section{INTRODUCTION}

Recently, pure electric vehicle have achieved sufficient driving performance due to important improvements in motors and batteries design technology. That, we can summarize the advantage of vehicle propelled by electric motor into the following point that the torque generation of an electric motor is very quick, accurate and can be measured easily [1].

In fact, the yaw rate of a car is influenced by disturbance torques resulting from crosswind, breaking, and acceleration on a -split road, so on, and a conventional front-wheel steering system cannot guarantee the vehicle stability on slippery roads. An electric vehicle equipped with two individual electric motors in the rear has the advantage of another steering control input, i.e. torque steering. Stability improvement, using torque steering is usually addressed as Direct Yaw-moment Control (DYC), $[2]$.

In this paper, we propose a hybrid Model Predictive Control (MPC) design that the aim is to track the above indicated reference, hence providing the driver with the desired yaw rate. The major advantage of MPC is the capability of handling in a single framework multiple inputs and outputs, constraints on inputs, states, and outputs, and optimization with respect to a predefined performance criterion.

In the next Section we formulate the vehicle dynamics using the front and rear tire slip angles as the states, and the vehicle yaw rate as the output. By assuming a constant longitudinal velocity and approximating the functions that relate the tire force to the tire slip angles by a piecewise affine maps, the vehicle dynamics are reformulated as a linear hybrid system in piecewise affine (PWA) form. By transforming the PWA model in an equivalent

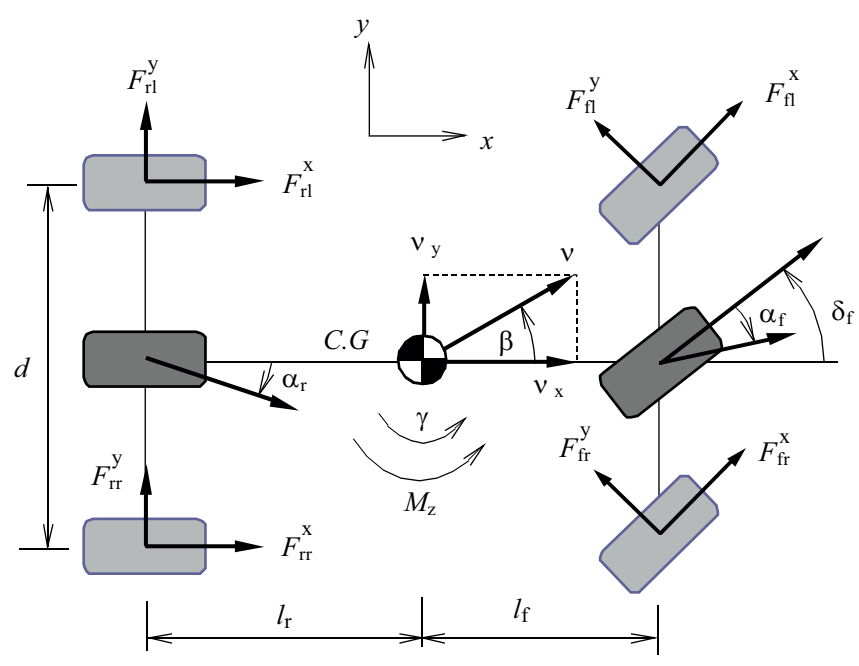

Fig. 1. Top-view vehicle dynamics model

\footnotetext{
* University of Science and Technology of Oran, Faculty of Electrical Engineering, BP 1505 El-Mnaouar, Oran, Algeria, khatir75@hotmail.com, bourah3@yahoo.fr, latyfo@hotmail.com
} 
mixed-logical dynamical (MLD) system, the obtained vehicle dynamics formulation is used as a prediction model in a hybrid model predictive control. For completeness the performance is investigated by simulations in last section, $[3]$.

\section{OVERALL SYSTEM MODELING}

\subsection{Vehicle model}

Figure (1) shows the planar model of an electric vehicle with two electric motors placed at the rear wheels. This vehicle model has two degree-of-freedom (DOF), ie, the yaw and lateral motions. However, the roll, vertical, and pitch motions are not considered here due to neglecting the suspension system. The load transfers induced by the lateral and longitudinal accelerations are also taken into account in the mathematical model [4]

- Lateral motion

$$
m v(\dot{\beta}+\gamma)=F_{y f l}+F_{y f r}+F_{y r l}+F_{y r r}
$$

- Yaw motion

$$
\begin{gathered}
I_{z} \dot{\gamma}=l_{f}\left(F_{y f l}+F_{y f r}\right)-l_{r}\left(F_{y r l}+F_{y r r}\right)+M_{z} \\
M_{z}=\frac{1}{2} d\left(F_{x r l}-F_{x r r}\right) \\
F_{f}=\left(F_{y f l}+F_{y f r}\right), \quad F_{r}=\left(F_{y r l}+F_{y r r}\right)
\end{gathered}
$$

where the longitudinal motion can be expressed as follow

$$
m \dot{v}=-\left(F_{x f l}+F_{x f r}+F_{x r l}+F_{x r r}\right)
$$

In the above equations, $m$ denotes the mass of the body, $I_{z}$ the moment of inertia concerning the yaw motion, $\beta$ the side slip angle, $\dot{\gamma}$ the yaw angular acceleration, $v$ the longitudinal velocity, $\delta_{f}$ the steering angle of front wheel, $l_{f}, l_{r}$ the distances from the center of gravity to the front and rear axles respectively, $M_{z}$ the yaw moment applied by differential braking, which must be determined from the control law.

\subsection{Electric Motor and Drive Modelling}

Permanent Magnet Synchronous (PMSM) motors are the most popular motors for in-wheel applications because typically they have a high power/weight ratio when compared with other machines and also have higher efficiency, since no power loss is associated with machine excitation. The developed torque of a salient pole PMS motor in $d-q$ coordinates is

$$
T_{d}=\frac{3}{2} p p\left(\lambda_{m} i_{q}-\left(L_{d}-L_{q}\right) i_{d} i_{q}\right)
$$

Furthermore, since the dynamic responses of modern motor drives are much faster than wheel dynamics, and considering the dominant poles of the closed loop system, an electric motor and its drive can be simply modeled as follows [5]

$$
G(s)=\frac{T}{T^{*}}=\frac{k}{\left(1+s T_{e}\right)\left(1+s T_{m}\right)},
$$

where $T_{e}$ is the delay due to inverter and $T_{m}$ is the electrical time constant of electric motor.

\section{CONTROL SYSTEM DESIGN}

In order to improve stability of the vehicle, the side slip angle and the yaw rate of the vehicle are controlled to trace their desired values. The direct yaw moment generated by the longitudinal forces is employed as the control input to make actual responses approach the desired values.

\subsection{Piecewise affine model}

The front and rear tire forces $F_{f}, F_{r}$ respectively, are nonlinear functions of the tire slip angles $\alpha_{s}, \alpha_{r}$ and of the longitudinal slip $\lambda \in[0,1]$.

Since the tire slip angles are small for high speed turns we can approximate $\tan (\alpha) \approx \alpha$, hence getting

$$
\alpha_{f}=\delta_{f}-\beta-\frac{l_{f} \gamma}{v}, \quad \alpha_{r}=-\beta+\frac{l_{r} \gamma}{v}
$$

We assume that during the turning maneuver the longitudinal velocity $\mathrm{v}$ is constant and we use a model of the tire forces which, for a constant longitudinal slip $s$, is piecewise linear

$$
\begin{aligned}
& F_{f}\left(\alpha_{f}\right)=\left\{\begin{array}{lrr}
-c_{f} \alpha_{f} & \text { if }-p_{f} \leqslant \alpha_{f} \leqslant p_{f} \\
-\left(d_{f} \alpha_{f}+e_{f}\right) & \text { if } & \alpha_{f}>p_{f}
\end{array}\right. \\
& F_{r}\left(\alpha_{r}\right)=\left\{\begin{array}{lrr}
-c_{r} \alpha_{r} & \text { if }-p_{r} \leqslant \alpha_{r} \leqslant p_{r} \\
-\left(d_{r} \alpha_{r}+e_{r}\right) & \text { if } & \alpha_{r}>p_{r}
\end{array}\right.
\end{aligned}
$$

where $c_{f}, c_{r}$ denote the cornering stiffness coefficients of the front and rear wheels respectively, $P_{f}$ and $P_{r}$ are called the critical side slip angle. The force equation (11) is simplified in order to reduce the complexity of the dynamical model. A qualitative sideslip angle-tire force characteristic is shown in Fig. 2. It is obvious from (11) that the tire forces are symmetric functions, ie, for any $j \in f, r$ and $\alpha_{j},[6]$

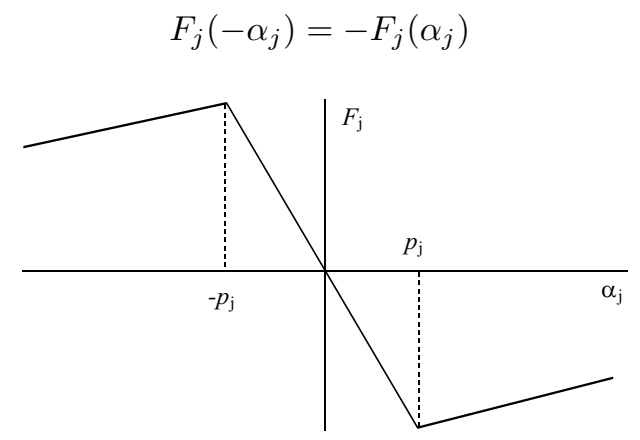

Fig. 2. Qualitative characteristic of the sideslip angle-tire force relation 


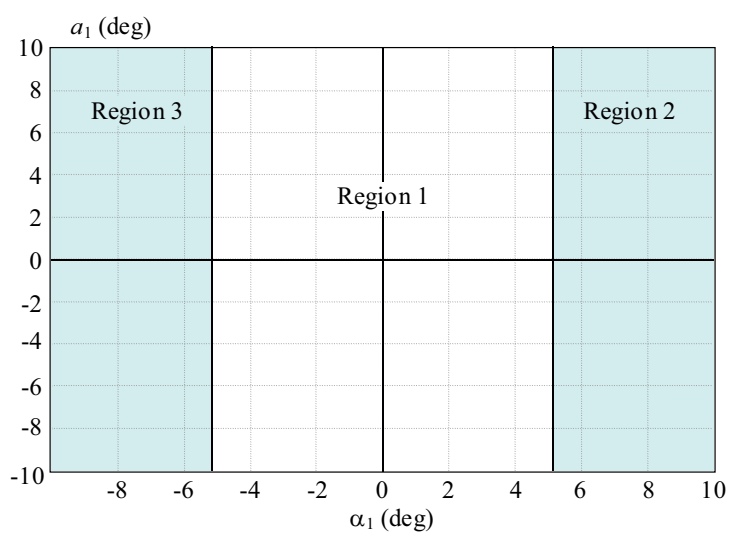

Fig. 3. Partitions adopted for the under steering vehicle model

We can now write the hybrid (PWA) bicycle model of the vehicle

$$
\begin{gathered}
{\left[\begin{array}{c}
\ddot{\beta} \\
\ddot{\gamma}
\end{array}\right]=\left[\begin{array}{cc}
-\frac{F_{f}\left(\alpha_{f}\right)+F_{r}\left(\alpha_{r}\right)}{m v} & -\frac{F_{f}\left(\alpha_{f}\right) a+F_{r}\left(\alpha_{r}\right) b}{m v}-v \\
-\frac{F_{f}\left(\alpha_{f}\right) a+F_{r}\left(\alpha_{r}\right) b}{I_{z} v} & -\frac{F_{f}\left(\alpha_{f}\right) a^{2}+F_{r}\left(\alpha_{r}\right) b^{2}}{I_{z} v}
\end{array}\right]\left[\begin{array}{c}
\dot{\beta} \\
\dot{\gamma}
\end{array}\right]} \\
+\left[\begin{array}{cc}
-\frac{F_{f}\left(\alpha_{f}\right)+F_{r}\left(\alpha_{r}\right)}{m} & 0 \\
-\frac{F_{f}\left(\alpha_{f}\right)+F_{r}\left(\alpha_{r}\right)}{I_{z}} & \frac{1}{I_{z}}
\end{array}\right]\left[\begin{array}{c}
\delta_{f} \\
M_{z}
\end{array}\right]
\end{gathered}
$$

The dynamics model is a second order piecewise affine (PWA) system (Sontag 1981), [7]

$$
\begin{gathered}
\dot{x}(t)=A_{i}^{c} x(t)+B_{i}^{c} u(t)+\varphi_{i}^{c} \\
y(t)=C^{c} x(t)+D^{c} u(t) \\
i \in I: H_{i} x(t) \leqslant K_{i}
\end{gathered}
$$

Where $x=\left[\beta,\left.y\right|^{\prime}\right.$ is the state vector, $u=\left[\delta_{f}, M_{z}\right]^{\prime}$ is the input vector, $y=\gamma$ is the output, and $i \in I$ is the active region, where $I=1 \dot{s}$ and $s$ is the number of regions of the PWA system. Inequalities (14c) are derived from the inequalities in (11), and partition the state space into polyhedral regions, that define the different operating conditions (linear, and positive and negative saturation, for each pair of tires). Hence, they are also called system modes. The matrices $A_{i}, B_{i}, i \in I, C, D$, define the vehicle dynamics in the different conditions, and are obtained by substituting the different force expressions (11) in (12). The active region (or active mode) $i$ of the PWA system is selected by evaluating (14c) for the current value of the state $x$.

Since (11) defines 3 conditions per each pair of tires, there are in total 9 modes. Since (12) is symmetric with respect to $\alpha_{j}$ due to the symmetry of (11) and the polyhedral partition is symmetric, the PWA vector field in (14a) is symmetric with respect to the state-input vector.

\subsection{Hybrid MPC designer}

Hybrid model predictive control has been recently applied to problems in automotive systems. In the MPC strategy, at each sampling instant a finite horizon open loop optimal control problem is solved, by using the current state as the initial condition.

Several modeling formalisms have been developed to describe hybrid systems, including PWA and MLD systems. The language HYSDEL (HYbrid Systems DEscription Language) was developed in [8] to obtain MLD models from a high level textual description of the hybrid dynamics. HYSDEL models are used in the Hybrid Toolbox for Matlab [9] for modeling, simulating, and verifying the safety properties of hybrid systems and for designing and prototyping hybrid MPC controllers. This section covers all the nonlinear elements experimented with during MPT control design [10]. These elements can be mixed and matched to investigate their combined effect on MPT controller performance and design is shows in Fig.6.

The piecewise affine model developed in Section III. A. is discretized in time with sampling period $T_{s}$

$$
\begin{gathered}
\dot{x}(k+1)=A_{i} x(k)+B_{i} u(k)+\varphi_{i} \\
y(k)=C x(k)+D u(k) \\
i: H_{i} x(t) \leqslant K_{i}
\end{gathered}
$$

Obtaining a model that can be used to design a hybrid MPC controller for tracking a reference yaw rate by actuating the active front steering and the differential braking.

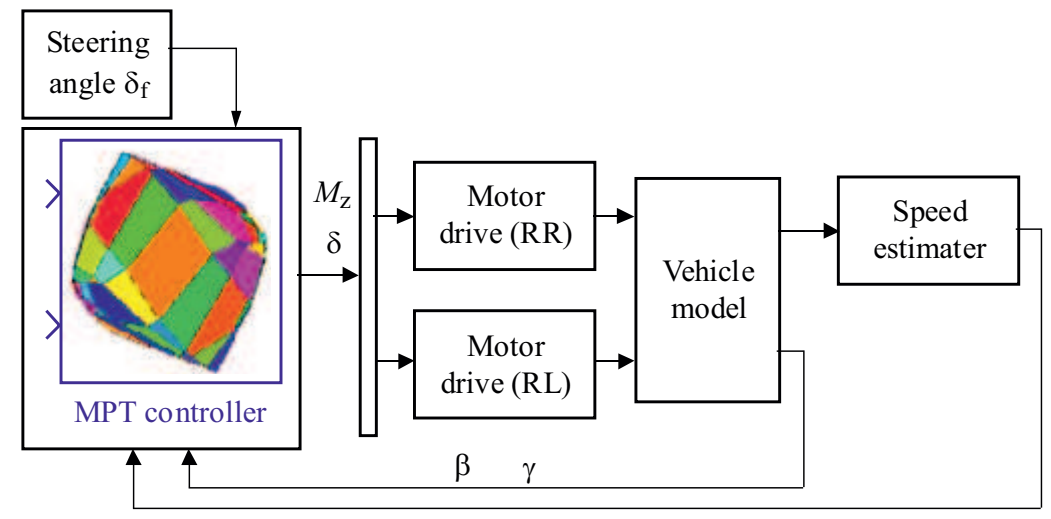

Fig. 4. General Scheme of the proposed control system with MPT control 


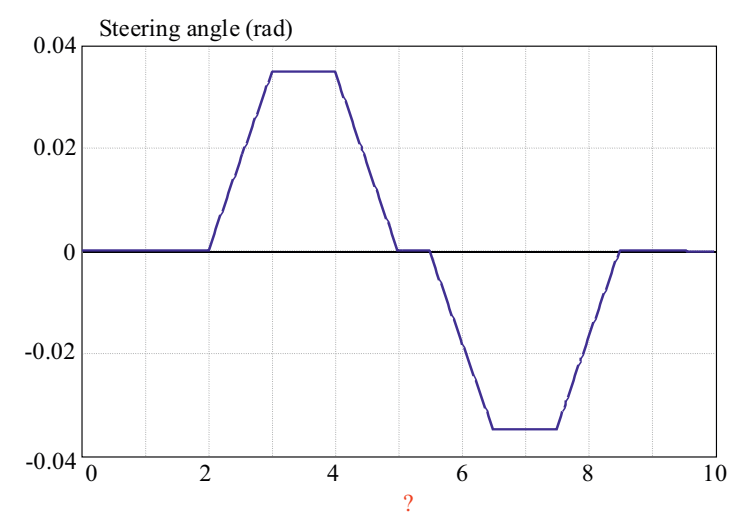

Fig. 5. Steering angle maneuvers

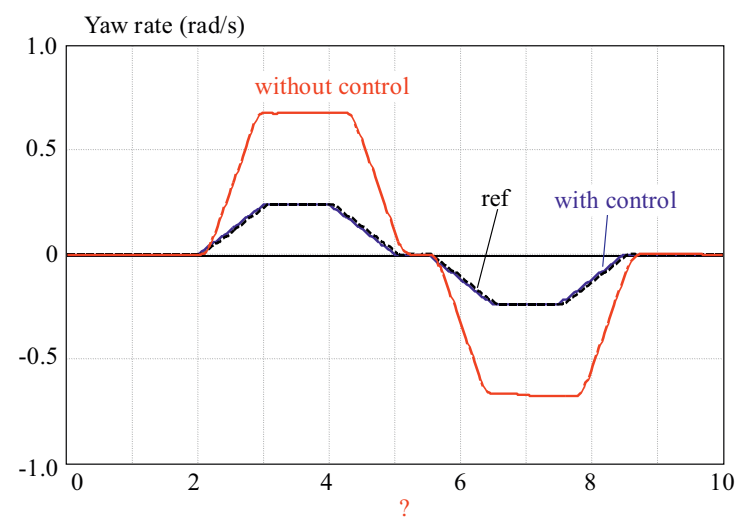

Fig. 7. Yaw rate and its reference in Close loop simulation

The design of such a controller has been explored by the authors in (Bernardini et al2009).

The hybrid MPC controller solves at every control cycle the problem

$$
\begin{gathered}
\min _{U_{n}(k)} j\left(x(k), U_{n}(k), \gamma_{r}\right) \\
\text { s.t. } \quad x\left({ }^{\prime} 0 \mid k\right)=x(k) \\
i(h \mid k): \quad H_{i(h \mid k)} x(h \mid k) \leqslant K_{i(h \mid k),} \\
x(h+1 \mid k): \quad(16 \mathrm{c}) \\
\left.A_{i(h \mid k)} x(h \mid k)+B_{i(h \mid k)} u(h \mid k)+\varphi_{i(h \mid k),}\right) \\
y(k)=C x(k)+D u(k) \\
u_{\min } \leqslant u(h \mid k) \leqslant u_{\max }, \\
x_{\min } \leqslant x(h \mid k) \leqslant x_{\max }, \\
y_{\min } \leqslant y(h \mid k) \leqslant y_{\max }, \\
h=0, \ldots N-1,
\end{gathered}
$$

Where $J$ is the (quadratic) cost function that encodes the control objective (yaw rate tracking), (16f),(16g),(16h) model the constraints on inputs, states, and outputs, $N$ is the prediction horizon and $U N(k)=u(0 \mid k), \ldots u(N-1 \mid k)$

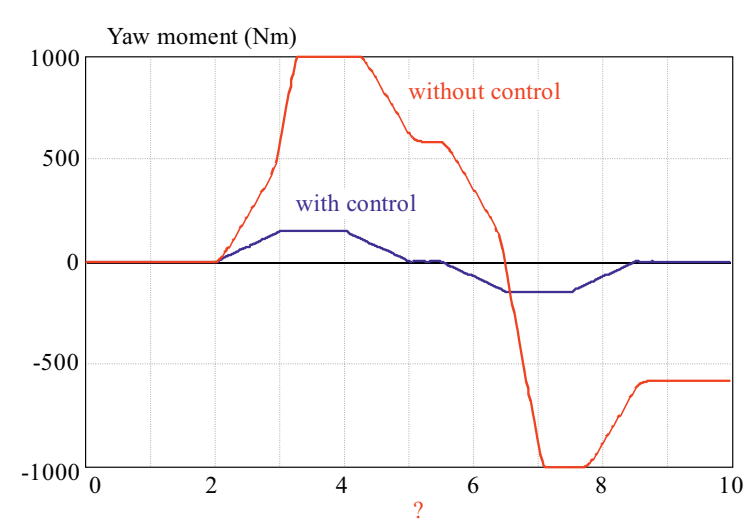

Fig. 6. Closed-loop actuation profiles yaw moment

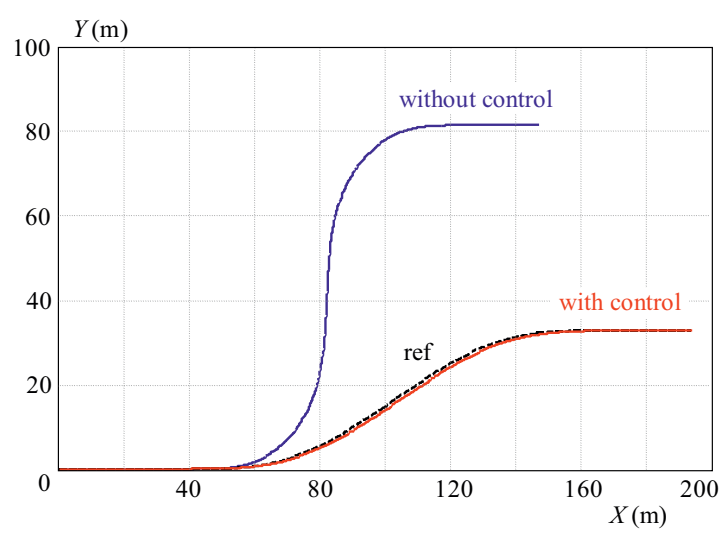

Fig. 8. Vehicle position during the simulation

\section{SIMULATION AND RESULTS}

In this section we show the simulation results for the MPC controller in closed loop with the nonlinear model of the vehicle dynamics which, besides the nonlinearities on the yaw rate and on the tire forces. However, the tire force curves of the simulation model match the one of the prediction model.

The nonlinear simulation model will not be described here in details, due to space limitations. We consider $m=1891 \mathrm{~kg}, I_{z}=3213 \mathrm{kgm}^{2}, a=1.47 \mathrm{~m}, b=1.43 \mathrm{~m}$, consistent with a typical passenger vehicle, and nominal speed $v=20 \mathrm{~m} / \mathrm{s}$. The parameters of the piecewise affine model of the tire forces are assumed $C_{f}=-9.06 \times 10^{4}$, $d_{f}=9.06 \times 10^{3}, e_{f}=-9.14 \times 10^{3}$ for the front tires, where the critical slip angle is $p_{f}=0.11 \mathrm{rad}$, and $C_{r}=$ $-1.65 \times 10^{5}, d_{r}=1.65 \times 10^{4}, e_{r}=-9.39 \times 10^{3}$ for the rear tires, with critical slip angle $p_{r}=0.06 \mathrm{rad}$. The switched MPC controller is simulated with sampling period $T_{s}=$ $50 \mathrm{~ms}$, and the horizons are $N=3$ steps for prediction.

The input constraints in (16f) are: $-0.35 \leq \delta \leq 0.35$ $\mathrm{rad},-1000 \leq M_{z} \leq 1000 \mathrm{Nm}$,

The state constraints in $(16 \mathrm{~g})$, which are due to the limited knowledge of the tire force maps, are: $-5 \leq \gamma \leq 5$ $\mathrm{rad} / \mathrm{s},-5 \leq y \leq 5 \mathrm{~m} / \mathrm{s}$.

The control scheme is depicted in Fig.4, the simulations focus on aggressive maneuvers shows in Fog.5, where changes abruptly as a square wave. 
Figure 7 shows that the controller keeps the vehicles yaw rate thoroughly near the yaw rate reference and that the yaw moment provided by differential braking is used during the transient, but it is zero at steady state, as required.

The braking torque on each wheel is obtained from yaw moment using the torque distribution scheme detailed in Fig. 4.

From Fig. 6 it can be observed that during this simulation the yaw moment it has a good control using MPT controller by local MPC and the controller stabilizes the system on a close steady state achievable yaw rate.

In Fig. 8, without the controller system, the vehicle passes the target lateral displacement and additional efforts are required to return the car into the path. Obviously the controller successfully helps the driver to handle the lane change.

\section{CONCLUSION}

In this paper, we have proposed a hybrid model predictive control approach for coordinating the active front steering and the electric control actuators affecting the dynamic stability of the vehicle. The formulation of a model based on the vehicle dynamics with respect to the front and rear tire slip angles, and the use of an approximation on the tire force characteristics that result into piecewise affine dynamics describing the overall complex vehicle system, the optimization problem of the MPC controller can be proposed as a mixed integer quadratic problem. The model formulation allows us to clearly analyze the stability region of the closed loop dynamics. Simulations in nominal and disturbed conditions have been shown, which suggest hybrid MPC as a promising and viable candidate for such a challenging and sensitive control issue in automotive engineering.

\section{Acknowledgement}

\section{REFERENCES}

[1] HORI, Y.: Future vehicle driven by electricity and Control-research on four-wheel-motored UOT electric march II, IEEE Transactions on Industrial Electronics 51 (2004), 954-962.

[2] N. Matsumoto, N.-TomizuKA, M.: Vehicle Lateral Velocity and Yaw Rate Control with two Independent Control Inputs, Proc. American Control Conference (1990), 1863-1875.

[3] BERNARDINI, D. DI-CAIRANOD. S.-BEMPORAD, A.TSENG, H. E.: Drive-by-wire Vehicle Stabilization and Yaw regulation: a Hybrid Model Predictive Control Design, Joint 48th IEEE Conference on Decision and Control and 28th Chinese Control Conference Shanghai, PR China, December 16-18, 2009.

[4] SHINO, M.-NAGAI, M. : Yaw-moment control of electric vehicle for improving handling and stability, JSAE Review 22 (2001), 473-480.
[5] TAHAMI, F. K. R.-FARHANGHI, S.-SAMADI, B. : Fuzzy Based Stability Enhancement System for a Four-Motor-Wheel Electric Vehicle, SAE Paper 2002-01-1588 (2002), 1825-1833.

[6] DI-CAIRANO, S.-TSENG, H. E.-BERNARDINI, D.-BEMPORAD, A. : Steering Vehicle Control by Switched Model Predictive Control, PUBLISHED WHERE, WHAT AS?

[7] SONTAG, E.: Nonlinear regulation: The piecewise linear approach, IEEE Trans. Automatic Control No. 26(2) (1981), 346-358.

[8] BEMPORAD, A.: Hybrid toolbox Users guide, 2003.

[9] GIORGETTI, N.-BEMPORAD, A.-TSENG, H.-HROVAT, D. : Hybrid model predictive control application towards optimal semi-active suspension, Int. J. Control 79 No. 5 (2006), 521-533.

10] KVASNICA, M.-GRIEDER, P.-BAOTIC, M-MORARI, M.: Multi Parametric Toolbox (MPT) In Hybrid Systems, Microelectronics Journal Computation and Control, Springer, 2003.

Received 19 May 2012

Khatir Tabti was born in Saida (Algeria) in 1975. He obtained a diploma of engineer in Electrotechnics in 1998. He received his master degree in electrical control engineering at University of Sciences and Technology of Oran (Algeria) from 2004 at 2006 . He is currently working toward the PhD degree at Mohamed Boudiaf University of Science and Technology of Oran. He works currently in an industrial company in Algeria as Head of Sustainable Development department. He is an associate lecturer at the University Moulay Tahar, Saida. His fields of interest include: Electrical drives control, Traction control system, dynamics control of electric vehicle.

Mohamend Bourahla was born in Naama, Algeria, in 1952. He received the Doctorate degree (Aspirantus) in Electrical Drives from the Technical University of Budapest, Hungaria and Doctorat d'Etat degree in Power Electronics from the University of Sciences and Technology of Oran (USTO), Algeria in 1983 and 1995, respectively. From 1983 to 1987 he teaches and conducts research in the area of Power Electronics and Electrical Drives at the Institute of Electrotechnics of USTO. From 1987 to 1991 he was a researcher at the University of Technical Sciences of Montpellier, France, where he conducts research work on GTO thyristor for electrical traction application. He is currently Professor at the Department of Electrotechnics of the University of Sciences and Technology of Oran, he is head of research laboratory of applied power electronics in Power Semi Conductor Devices, Powers Electronics Structures (PES) in applications of Electrical traction, solar energy, Telecommunication and Medical.

Lotfi Mostefai received his master degree in Control Engineering in 2002, and a PhD degree in 2010 in Automatic at at Mohamed Boudiaf University of Science and the Technology of Oran (USTMB). He has been giving lectures on Control Engineering, Microprocessors and Microcontrollers in Industrial applications and Instrumentation in Biomedical Engineering since 2002 at the University Moulay Tahar in Saida (UTMS), Algeria. He is currently a member of Genie Electrotechnics Laboratory in UTMS His interests are mainly on: optimal and robust control, motion control systems, smart materials and their application, automotive engineering and renewable energies. 